\title{
Susanne Günthner
}

\section{Wenn-Konstruktionen im Gespräch: Zur Verwobenheit kognitiver und interaktionaler Faktoren bei der Realisierung grammatischer Muster}

\begin{abstract}
Auf der Basis einer empirischen Analyse von wenn-Konstruktionen im gesprochenen Deutsch wird verdeutlicht, wie und mit welchen Funktionen Sprecherinnen und Sprecher unterschiedliche wenn-Konstruktionen im kommunikativen Handeln aktualisieren. Die Analyse, die sich an der interaktional ausgerichteten Konstruktionsgrammatik (Günthner \& Imo 2006; Imo 2007; Günthner, Imo \& Bücker 2014; Bücker, Günthner \& Imo 2015) ausrichtet, veranschaulicht, dass Interagierende über eine Bandbreite an wenn-Konstruktionen mit unterschiedlichen Graden syntaktischer, prosodischer, sequenzieller und handlungsbezogener Integration zwischen dem subordinierten wenn-Teil und der Folgeeinheit verfügen. Folglich zeigt sich nicht nur ein differenzierteres Bild von grammatischen Konstruktionen, als die Vorstellung einer statischen 1:1-Form-Funktions-Paarung zulässt, sondern die kommunikativen Gebrauchsweisen weisen darüber hinaus auf eine enge Verwobenheit kognitiver und interaktionaler Prinzipien bei der Realisierung von grammatischen Konstruktionen im konkreten Interaktionsvorgang hin. Die Vielfalt der in Korpora gesprochener Daten vorfindlichen Varianten von wenn-Konstruktionen stellt somit die Annahme von Konstruktionen als isolierte Schemata in Frage und veranschaulicht stattdessen, wie flexibel Sprecherinnen und Sprecher grammatische Konstruktionsbausteine im Vollzug ihres kommunikativen Handelns den jeweiligen Interaktionsprozessen anpassen.
\end{abstract}

\section{Einleitung}

Empirische Arbeiten zum Gebrauch grammatischer Strukturen verdeutlichen immer wieder, dass die Realisierung syntaktischer Konstruktionen in enger Ver-

Ich danke Jörg Bücker, Verena Wecker sowie den anonymen Gutachterinnen und Gutachtern für ihre Kommentare.

Susanne Günthner, Germanistisches Institut, Westfälische Wilhelms-Universität Münster

Ә Open Access. (c) 2021 Susanne Günthner, publiziert von De Gruyter. (cc) BY Dieses Werk ist lizensiert unter einer Creative Commons Namensnennung 4.0 International Lizenz. https://doi.org/10.1515/9783110710595-006 
bindung mit den jeweiligen kontextbezogenen Aktivitäten im Prozess der Interaktion eingesetzt wird (Auer \& Pfänder 2011; Günthner, Imo \& Bücker 2014; Bücker, Günthner \& Imo 2015; Deppermann \& Günthner 2015). Doch trotz ihrer situativen Emergenz und lokalen Anpassung erweisen sich grammatische Strukturbildungen keineswegs als beliebig aushandelbar, sondern sie gründen auf Sedimentierungen, die unterschiedliche Ebenen - prosodische Gestaltung, lexikalische Kookkurrenzen, sequenzielle Platzierung etc. - umfassen. Diese haben sich im Verlauf einer langen Kette von Interaktionssituationen verfestigt und sind somit Bestandteil des sprachlichen Wissensvorrats der Gesprächsteilnehmerinnen und Gesprächsteilnehmer: ${ }^{1}$

Wenn Menschen wiederholt „ähnliche“ Dinge in „ähnlichen“ Situationen sagen, entwickelt sich daraus mit der Zeit ein sprachliches Verwendungsmuster, das in den Köpfen der Benutzer als neue Kategorie oder Konstruktion schematisiert wird - mit unterschiedlichen Abstraktionsgraden.

(Tomasello 2006: 21)

Die relative Stabilität sprachlicher Muster stellt geradezu eine notwendige Bedingung für das Gelingen sprachlicher Interaktion dar (Luckmann 2002: 208; Günthner 2009, 2011a, 2011b): Bei der Durchführung sprachlicher Handlungen orientieren sich Interaktionsteilnehmerinnen und Interaktionsteilnehmer an diesem „structured inventory of conventional linguistic units“ (Langacker 1987: 57); zugleich werden diese Muster im jeweiligen Interaktionsprozess situativ re-kontextualisiert und den Gegebenheiten im ,hic et nunc' der Interaktion angepasst (Günthner 2011b). Somit entsteht - wie u. a. Arbeiten zur Grammatikalisierung und Emergence Grammar verdeutlichen (Hopper \& Traugott 1993, 2003; Hopper 1987, 1988, 2004; Günthner \& Hopper 2010; Auer \& Pfänder 2011) - eine fortwährende Spannung zwischen Sedimentierung und Emergenz bzw. zwischen „convention and usage“ (Langacker 1987: 70).

Die vorliegende Analyse veranschaulicht, wie und mit welchen Funktionen Sprecherinnen und Sprecher unterschiedliche wenn-Konstruktionen im kommunikativen Handeln aktualisieren. In Anlehnung an die interaktional ausgerichtete Konstruktionsgrammatik (Günthner \& Imo 2006; Imo 2007; Günthner, Imo \& Bücker 2014; Bücker, Günthner \& Imo 2015; Günthner 2009, 2011a, 2011b, 2015a) werden grammatische Konstruktionen hierbei keineswegs als dekontextualisierte und auf die Einheit eines - an der Schriftsprache orientierten - Satzes reduzierte Strukturen untersucht, ${ }^{2}$ sondern als merkmalsreiche, sequenziell organisierte und kontextkontingente Ressourcen zur Bewältigung

1 S. auch Bittner \& Köpcke (2007) zu grammatischem Wissen (am Beispiel der Verbmorphologie). 2 S. Ágels (2003) Kritik am Skriptizismus der Grammatikschreibung. 
spezifischer kommunikativer Aufgaben (Günthner 2011a, 2011b). Grammatische Konstruktionen haben somit - wie Bücker (2015: 453) ausführt - „große Ähnlichkeiten mit den in der kognitiv-funktionalen Morphologie Bybees diskutierten Schemata, die sprachsystemgebundene, gestalthafte Abstraktionen ausdrucksseitiger Familienähnlichkeiten zwischen unterschiedlichen sprachlichen Zeichen innerhalb einer bestimmten grammatischen Funktionsklasse repräsentieren“. So stellen Konstruktionen bzw. Schemata „gebrauchsbasiert geprägte sprachsystemspezifische Einheiten“ dar, die „als kognitiv und sozio-kulturell reale Phänomene bzw. natürliche Kategorien zum situationstranszendenten Sprachgebrauchswissen der Interagierenden gehören“ (Bücker 2015: 453). ${ }^{3}$

Wie die folgende Analyse zeigt, verfügen Interagierende in der kommunikativen Praxis über eine Bandbreite an wenn-Varianten - mit unterschiedlichen Graden prosodischer, syntaktischer, semantischer, sequenzieller und handlungsbezogener Integration zwischen dem subordinierten wenn-Teil und der Folgeeinheit -, die allesamt Teil des Wissenshaushalts (Luckmann 2002) und damit des „situationstranszendenten Sprachgebrauchswissens“ (Bücker 2015: 453) sind und zugleich lokal gehandhabt werden. ${ }^{4}$ Zugleich verweisen die vorliegenden Daten auf die enge Verwobenheit kognitiver und interaktionaler Prinzipien bei der Realisierung dieser grammatischen Konstruktionen im Alltagsgebrauch.

Die Analyse basiert auf einem Korpus von 90 Gesprächen (mit einer Dauer von 30 bis 180 Minuten), die zwischen 1989 bis 2017 in verschiedenen Gegenden Deutschlands aufgezeichnet wurden: Das Korpus enthält informelle faceto-face- und Telefon-Gespräche unter Freundinnen und Freunden, Bekannten und Familienangehörenden, sowie universitäre Sprechstundengespräche, ArztPatienten-Interaktionen, Radio-Phone-Ins, Talkshows und Reality-TV-Sendungen.

\section{Wenn-Konstruktionen im Gebrauch}

\footnotetext{
My chief object in writing this chapter has been to make the reader realize that language is not exactly what a one-sided occupation with dictionaries and the usual grammars might lead us to think, but a set of habits, of habitual actions, and that each word and each sentence spoken is a complex action on the part of the speaker. The greater part of
}

3 S. auch Einleitung (i.d.B.: 2) sowie Bittner (i.d.B.) zu usage-based Ansätzen der Construction Grammar.

4 Zur Debatte um die Vielzahl formaler Varianten von wenn-Realisierungen und der Frage der Identität von Konstruktionen s. Deppermann (2011). 
these actions are determined by what he has done previously in similar situations, and that again was determined chiefly by what he had habitually heard from others. But in each individual instance, apart from mere formulas, the speaker has to turn these habits to account to meet a new situation to express what has not been expressed previously in every minute detail; therefore he cannot be a mere slave to habits, but has to vary them to suit varying needs - and this in course of time may lead to new turns and new habits; in other words, to new grammatical forms and usages.

(Jespersen 1924: 29)

\subsection{Syntaktisch integrierte wenn-Konstruktionen}

Wenn-Konstruktionen (wie „wenn Jürgen kommt, spiele ich nicht mit“5) gelten traditionellerweise als komplexe Satzgefüge bzw. als biklausale Konstruktionen mit zwei Teilsätzen, die aus zwei Propositionen - einem subordinierten wennSatz (der Protasis „p“; z. B. „wenn Jürgen kommt“) und einem Matrixsatz (der Apodosis bzw. Konsequenz „q“; z. B. „spiele ich nicht mit“) - bestehen. Logisch betrachtet können „p“ und „q“ wahr, „p“ falsch und „q“ wahr, oder aber „p“ und „q“ falsch sein. Ausgeschlossen ist lediglich die Möglichkeit, dass „p“ wahr und „q“ falsch ist (Comrie 1986). Doch wie u. a. Sweetser (1990: 115-116) verdeutlicht, haben (prototypische) Konditionalsätze in der Alltagsverwendung häufig die Lesart „,if and only if“ (,iff“), d.h. die Protasis wird nicht nur als hinreichende, sondern als notwendige Bedingung für die Apodosis interpretiert (d. h. „nur wenn Jürgen kommt, spiele ich nicht mit“).

Obgleich der wenn-Satz dem Matrixsatz sowohl vorausgehen (,Wenn Jürgen kommt, spiele ich nicht mit“), als auch folgen („Ich spiele nicht mit, wenn Jürgen kommt“) kann, ${ }^{6}$ zeigen empirische Studien zum gesprochenen Deutsch, dass der wenn-Teilsatz (und damit die Protasis) meist vorangestellt ist und einen Hauptsatz (die Apodosis) projiziert (Günthner 1999, 2012; Auer 2000; Wegner 2010; Auer \& Lindström 2015). ${ }^{7}$ Im Sinne der Kognitiven Linguistik fungiert der wenn-Satz bzw. die Protasis als „space-builder“, der einen „mental space“ (Fauconnier 1985; Sweetser 1990) aufbaut, über den dann in der Apodosis etwas ausgesagt wird. Erst mit der Produktion des Matrixsatzes gilt diese zweiteilige Konstruktion als vollständig.

5 Dieses Beispiel entstammt Köpcke/Panther (1989: 686).

6 In seltenen Fällen werden wenn-Sätze auch parenthetisch in ein Satzgebilde einbettet („Ich spiele, wenn Jürgen kommt, nicht mit“), wobei sie die Produktion des Hauptsatzes unterbrechen (vgl. Auer 2000).

7 In den vorliegenden Daten sind 58\% der wenn-Sätze präpositioniert, 31\% dagegen postpositioniert. $11 \%$ sind entweder alleinstehende wenn-Konstruktionen, wenn-Fragmente oder unklare Fälle (,false starts“, Reformulierungen etc.). 
Der folgende Ausschnitt illustriert einen solchen scheinbar „prototypischen“ Gebrauch einer biklausalen wenn-Konstruktion:

(1) REISE NACH SALZBURG (NRW 2008) ${ }^{8}$

019 Bel: [und ]

$020 \quad \mathrm{jA}$ ?

$021 \quad(0.5)$

022 Lea: also wenn du_s geMüTlich haben willst-

023 n_nimmst du den ZUG.

$024 \quad(1.0)$

025 Bel: [OKAY.]

Lea beginnt in Zeile 022 ihren Redezug mit dem Diskursmarker „also“, gefolgt von einem wenn-Teilsatz („wenn du_s geMÜTlich haben willst-“; Z. 022), dessen prosodische Kontur den Nukleusakzent auf „MÜT“ hat und mit einem gleichbleibenden Tonhöhenverlauf (,willst-“) endet. Der wenn-Teil (Protasis) baut hier den „mentalen Raum“ auf, über den dann in der Apodosis etwas ausgesagt wird. Er bildet also keine abgeschlossene syntaktische Gestalt, sondern projiziert prosodisch, syntaktisch wie auch semantisch und pragmatisch eine Fortsetzung: Erst mit der Produktion der erwartbaren Konsequenz „n_nimmst du den ZUG.“ (Z. 023) ist das zweiteilige Satzgebilde abgeschlossen. Beide Konstruktionsteile indizieren eine enge syntaktische Integration:

i. Der wenn-Teilsatz, der Verbendstellung (,willst-“) und somit eine subordinierende Satzstellung aufweist, bildet die im Vorfeld positionierte Konstituente innerhalb der Topologie des Gesamtsatzes:

\begin{tabular}{|c|c|c|c|c|}
\hline Vorfeld & $\begin{array}{l}\text { linke } \\
\text { Satzklammer }\end{array}$ & Mittelfeld & $\begin{array}{l}\text { rechte } \\
\text { Satzklammer }\end{array}$ & Nachfeld \\
\hline
\end{tabular}

wenn du_s geMÜTlich n_nimmst du den ZUG.

haben willst-

ii. Der durch den wenn-Teilsatz projizierte Hauptsatz setzt mit dem finiten Verb „nimmst“ ein (Inversionsstellung) ${ }^{9}$, was auf eine Integration des vorausgehenden subordinierten Teilsatzes hinweist. (Gelegentlich werden Hauptsätze auch mit einem resumptiven Element - wie dann, so etc. - eingeleitet.)

8 Die Transkripte orientieren sich an den GAT 2-Konventionen (Selting et al. 2009).

9 Hierzu auch Köpcke/Panther (1989). 
Biklausale wenn-Konstruktionen können prosodisch sowohl integriert sein (d. h. Protasis und Apodosis werden unter einem prosodischen Dach realisiert), als auch eigenständige prosodische Konturen (wie im vorliegenden Ausschnitt (1) REISE NACH SALZBURG) bilden. Wie Ono \& Thompson (1995: 221) ausführen, erweist sich die Realisierung syntaktischer Schemata „[as] both locally managed and extremely dynamic“; d. h. der konkrete interaktive Gebrauch grammatischer Schemata ist insofern kontextkontingent, als „the realization of these schemas depends heavily on needs and constraints arising at the moment of speech in the situated interactional events“ (Ono \& Thompson 1995: 221). Folglich verwundert es nicht, dass Sprecherinnen und Sprecher im gesprochenen Deutsch neben der als standardsprachlich kodifizierten syntaktisch integrierten wenn-Konstruktion über eine Vielzahl weiterer wenn-Formate verfügen: Diese beinhalten wenn-Strukturen mit syntaktischer, semantischer und prosodischer Integration wie auch Nicht-Integration (auf sämtlichen Ebenen), sowie komplexe, mehrere Turnkonstruktionseinheiten (TCUs) umfassende Segmente. Ferner verfügen sie über verdichtete, fragmentarische wenn-Einheiten sowie über insubordinierte, alleinstehende wenn-Sätze. Diese unterschiedlichen wenn-Formate setzen Sprecherinnen und Sprecher - wie die vorliegende Analyse zeigt - je nach „needs and constraints arising at the moment of speech in the situated interactional events“ (Ono \& Thompson 1995: 221) ein.

\subsection{Syntaktische (und prosodische) Nicht-Integration des initialen wenn-Satzes}

Sprecherinnen und Sprecher im gesprochenen (und teilweise geschriebenen) Deutsch verwenden immer wieder initial positionierte wenn-Teilsätze, die insofern vom schriftsprachlichen Standard abweichen, als ihnen ein Matrixsatz folgt, der - wie Köpcke \& Panther (1989: 687) ausführen - keine Inversion aufweist: „[. . .] the constituent order in the consequent clause is the same as that found in a simple declarative sentence“. Der Matrixsatz markiert somit keine syntaktische Anbindung an den vorausgehenden subordinierten wenn-Teilsatz. ${ }^{10}$ Die Prosodie stützt die syntaktische Unabhängigkeit insofern, als sich diese „nicht-integrierten“ Konstruktionen aus eigenständigen Intonationseinheiten mit eigenen Nukleusakzenten zusammensetzen.

10 Zu syntaktisch „abweichenden“ wenn-Sätze siehe auch Weinrich (1993/2007: 740); Helbig/ Buscha (2005: 576f.); Wegner (2010). Vgl. auch Zifonun et al. (1997: 2290) zu „moduskommentierenden“ Konditionalsätzen. 
In den Daten zeichnen sich allerdings verschiedene Typen syntaktisch nichtintegrierter wenn-Konstruktionen ab: (a) nicht-integrierte wenn-Konstruktionen, die in eine integrierte Wortstellung konvertierbar sind, und (b) nicht-integrierte wenn-Konstruktionen, die nicht in eine integrierte Wortstellung konvertierbar sind.

\section{a. Nicht-integrierte wenn-Konstruktionen, die in eine integrierte Wortstellung konvertierbar sind}

Der folgende Ausschnitt entstammt einem Gespräch unter Freundinnen:

(2) HEIRATEN (NRW 2012)

121 Ina: un_un waRUM nich?

122 Kira: wenn wir heiraten WOLLt [en, ]

123 Meike: [hm ]

124 Kira: <<kichernd> w_wir hätten es LÄNGST getAn;>

125 Ina: <<kichernd> ECHT. hihihi[hihihi]

126 ?: [

Kiras Äußerung setzt mit einem initialen wenn-Teilsatz „wenn wir heiraten WOLLt[en,]“ (Z. 122) ein, der weder syntaktisch, semantisch noch prosodisch vollständig ist und folglich eine Fortsetzung - die Konsequenz - erwartbar macht. Diese zeigt allerdings keine „TOP-V-Stellung“ (Köpcke \& Panther 1989), sondern sie wird asyndetisch realisiert: „<<kichernd> w_wir hätten es LÄNGST getAn;“; Z. 124). Die prosodische Gestaltung unterstützt die Nicht-Integration insofern, als der ,space-builder“ „wenn wir heiraten WOLLt[en,]“ mit dem Nukleusakzent auf „WOLL“ eine eigene Kontur bildet. Auch der Folgeteil (die Konsequenz) bildet eine prosodische eigenständige Einheit.

Der wenn-Teilsatz besetzt hierbei nicht länger die Vorfeld-Position, sondern hat die Position des Vor-Vorfelds inne:

\begin{tabular}{llllll}
\hline Vor-Vorfeld & Vorfeld & $\begin{array}{l}\text { linke } \\
\text { Satzklammer }\end{array}$ & Mittelfeld & $\begin{array}{l}\text { rechte } \\
\text { Satzklammer }\end{array}$ & Nachfeld \\
\hline $\begin{array}{l}\text { wenn wir heiraten } \\
\text { WOLLt[en, }]\end{array}$ & w_wir & hätten & es LÄNGST & getan; & \\
\hline
\end{tabular}

Trotz der aggregativen syntaktischen Realisierung liegt hier eine ,inhaltsbezogene Konditionalität“ („content conditionality“; Köpcke \& Panther 1989) vor, 
und die wenn-Konstruktion wäre problemlos in die als prototypisch geltende Satzstellung umstellbar: „wenn wir heiraten WOLLt[en,], hätten w_wir es LÄNGST getan;“.

\section{b. Nicht-integrierte wenn-Konstruktionen, die nicht in eine integrierte Wortstellung konvertierbar sind}

Wie bereits bei Köpcke \& Panther (1989) erwähnt, so finden sich auch im vorliegenden Korpus gesprochener Alltagsgespräche nicht-integrierte wenn-Äußerungen, die keine „inhaltsbezogene Konditionalität“ ausdrücken und folglich auch nicht in eine integrierte Wortstellung konvertierbar sind.

Ausschnitt (3) entstammt einer TV-Talkshow mit Pfarrer Fliege zum Thema Heimkinder. Der Sprecher Uwe, ein ehemaliges Heimkind, beschreibt, wie er seine früheren Mitschüler wahrgenommen hat:

(3) HEIMKINDER (2005_07_21kinderheim)

423 Uwe: dort; (-)

424 also- $\ddot{\mathrm{A}},($. )

425 was einen nicht UMbringt,

426 macht einen STARK, (.)

427 UND, (--)

428

429

430

431

432

wenn ich dann zum BEIspiel (an) meine-

433

434 ä_meine MITschüler: : ; (-)

435

436 wieder aus AUgsburg geTROFFen hab; (.) ja DEnen hat noch mit-

(1.5)

437 Uwe: die Unterhosen [irgendwo] hingeLEGT, $(0.5)$

439

hm: in_nem letzten SCHULjahr,

440 Uwe: daha (. ) ich hab immer dieSELbe angezogen;

Der Sprecher setzt in Zeile 428 mit dem subordinierten Teilsatz „wenn ich dann zum BEIspiel (an) meine- ä_meine MITschüler::; (-) wieder aus AUgsburg geTROFFen hab; (.)“ zu einer komplexen wenn-Konstruktion an. Der Matrixsatz wird auch hier asyndetisch angereiht: ,ja DEnen hat noch mit- (1.5) hm: in_nem 
letzten SCHULjahr, vorm abiTUR- die mUtter das pAUsebrot geSCHMIE:RT, die Unterhosen [irgendwo] hingeLEGT,“ (Z. 431-437). Doch im Unterschied zur vorangegangenen nicht-integrierten wenn-Konstruktion (Ausschnitt (2) HEIRATEN) handelt es sich hier nicht um eine traditionelle bzw. propositionale Konditionalverknüpfung: Der Matrixsatz liefert keineswegs die Konsequenz, die aus der Aussage in der Protasis folgt; vielmehr ist die Aussage im Matrixsatz (,ja DEnen hat noch mit- (1.5) hm: in_nem letzten SCHULjahr, vorm abiTUR- die mUtter das pAUsebrot geSCHMIE:RT, die Unterhosen [irgendwo] hingeLEGT,“; Z. 431-437) wahr bzw. gültig, unabhängig davon, ob Uwe diese Mitschüler getroffen hat oder nicht. ${ }^{11}$ Folglich ist die vorliegende Konstruktion auch nicht (ohne Bedeutungsänderung) in eine syntaktisch integrierte Satzstellung konvertierbar: „wenn ich meine Mitschüler aus Augsburg wieder getroffen hab, (dann) hat denen die Mutter das Pausenbrot geschmiert, die Unterhosen hingelegt“. ${ }^{12}$

Im nächsten Ausschnitt berichtet Mia ihrer Kollegin Anne, dass Leo, ein Ex-Kommilitone, sich gemeldet hat, um während einer Tagung in X-Stadt bei ihr zu übernachten:

(4) TAGUNG IN X-Stadt (2000)

228 Mia: un ehm wollte bei bei UNS übernAchten;

229 Ann: äh?

230 alter SCHNORrer (.) [EH. ]

231 Mia: [hab]

232 (ihm) dann auch sofort GSAGT,

$233 \quad(0.2)$

234 hihi $<<f>$ wenn du_n BETT brauchst,

235 hast du nIch en BRUder in X-stadt; $>$

236 Ann: [klar ] HAT_er.

237 Mia: [(oder)]

Beim vorliegenden wenn-Syntagma (Z. 234-235) handelt es sich um keine insitu-Realisierung, sondern um eine in Form von Redewiedergabe rekonstruierte Äußerung der Sprecherin Mia. Der durch Kicherpartikeln „hihi“ eingeleitete wenn-Teilsatz „,< $<>$ wenn du_n BETT brauchst,“ (Z. 234), der mit einer leicht steigenden Intonationskontur und erhöhter Lautstärke realisiert wird, die eine

$11 \mathrm{Zu}$ Wahrheitsbedingungen in Konditionalsätzen s. Günthner (1999).

12 S. Köpcke/Panther (1989: 688-690) zu typischen Hauptsatzphänomen-Tests (wie syntaktische Einbettung, Topikalisierung in der Apodosis, „left dislocation“, „consecutio temporum“), die bei den vorliegenden nicht-konvertierbaren wenn-Konstruktionen nicht funktionieren. 
Fortsetzung projizieren, fungiert als Hintergrund für Mias folgende (rhetorische) Frage: „ $<<\mathrm{f}>$ hast du nIch en BRUder in X-stadt; >“.

Auch hier initiiert der wenn-Teil keine traditionelle Konditionalbeziehung: Die dem wenn-Teil folgende Fragehandlung , $<<\mathrm{f}>$ hast du nIch en BRUder in Xstadt; > “ (Z. 235) bildet nicht die Konsequenz aus der im wenn-Teil produzierten Information. Stattdessen handelt es sich bei der wenn-Konstruktion um eine von Johnson-Laird (1986) und Köpcke \& Panther (1989) als „relevance conditional“ bezeichnete Relation: ${ }^{13}$ Die Sprecherin / der Sprecher impliziert, dass die Information in der Apodosis für das Gegenüber relevant ist, falls die Aussage in der Protasis zutreffen sollte: „if $\mathrm{p}$ is true, then the speech act expressed by q is relevant“ (Köpcke \& Panther 1989: 699). Die in den Gesprächsausschnitten (3) HEIMKINDER und (4) TAGUNG IN X-STADT verwendeten wenn-Einheiten

- „wenn ich dann zum BEIspiel (an) meine- ä = meine MITschüler::; (-) wieder aus AUgsburg geTROFFen hab;“

- „ $\ll<\mathrm{f}>$ wenn du_n BETT brauchst,“

zeigen deutliche Überschneidungen mit „Projektorkonstruktionen“ (Günthner 2008a, 2008b; 2011a, 2011b; Hopper \& Thompson 2008; Günthner \& Hopper 2010; Pekarek Doehler 2011), die eine zentrale Rolle bei der kognitiven und interaktionalen Architektur kommunikativer Ergebnisse innehaben: Diese initial positionierten wenn-Einheiten tragen insofern zum online-Management interaktionaler Handlungen bei, als sie die betreffenden Folgehandlungen (seien dies Aussage- oder aber Fragesätze) rahmen und so die Inferenzen der Rezipientinnen und Rezipienten leiten. ${ }^{14}$

Darüber hinaus veranschaulichen die präsentierten Gesprächsausschnitte (3) HEIMKINDER und (4) TAGUNG IN X-STADT, dass wenn-Teilsätze zwar eine Fortsetzung (Matrixsatz) erwartbar machen, die formale Ausgestaltung der projizierten Folgeeinheit allerdings unbestimmt bleibt: Während im Ausschnitt (3) dem wenn-Teil ein Aussagesatz folgt, leitet der wenn-Teil in (4) einen Fragesatz ein. Wie Auer (1996: 320) betont, sind syntaktische Projektionen keineswegs mit Determinationen gleichzusetzen: Sprachliche Muster bzw. Schemata sind gebrauchsbasiert geprägte Einheiten, die lokal im Prozess der Interaktion aktualisiert werden und somit eine gewisse Offenheit und Flexibilität aufweisen.

\footnotetext{
13 Neben „relevance conditionals“ (Johnson-Laird 1986; Köpcke/Panther 1989) werden diese wenn-Konstruktionen auch als „speech act conditionals“ (Sweetser 1990) bezeichnet. Hierzu ausführlich Günthner (1999).

14 Auch wenn diese einleitenden wenn-Teile - vergleichbar mit „Introgliedern“ (Ágel 2017: 92) - eine Folgeeinheit projizieren, sind sie idiomatisch nicht fixiert (wie bei „wenn ich ehrlich bin . . . “, „ohne Scherz . . . " etc. der Fall).
} 


\subsection{Kollaborativ erzeugte wenn-Konstruktionen}

Eines der zentralen Charakteristika von Alltagsgesprächen ist deren inhärente Dialogizität. Die intersubjektive Koordination sprachlichen Handelns im Hier-undJetzt der Interaktion ist deshalb möglich, weil die Prozesse sprachlicher Produktion und Rezeption in der Face-to-Face-Kommunikation maximal synchronisiert sind (Schütz 1932/73; Günthner 2015b): Gesprächsteilnehmerinnen und -teilnehmer überwachen den Gesprächsablauf, passen ihre Beiträge entsprechend an und erzeugen auf diese Weise gemeinsames sprachliches bzw. soziales Handeln. Diese Ausrichtung am Gegenüber hat zugleich erhebliche Auswirkungen auf grammatische Strukturbildungen: So werden grammatische Gestalten oftmals von mehreren Gesprächsteilnehmerinnen und -teilnehmern ko-konstruiert (Szczepek 2000a, 2000b; Benning 2012; Dausendschön-Gay, Gülich \& Krafft 2015). Gerade für die kognitive Linguistik und die gebrauchsbasierten Ansätze der Konstruktionsgrammatik sind solche von mehreren Sprecherinnen und Sprecher kollaborativ erzeugte Gestalten insofern aufschlussreich, als sie nicht nur die Emergenz grammatischer Strukturbildung im Prozess der Interaktion beleuchten, sondern wesentliche Hinweise auf intersubjektiv geteiltes Wissen um grammatische Schemata liefern (Günthner 2015a, 2015b, 2020).

Im Folgenden werde ich zwei Gesprächsausschnitte mit kollaborativ erzeugten, syntaktisch integrierten wenn-Konstruktionen präsentieren. ${ }^{15}$ Der erste Ausschnitt entstammt einem Gespräch zwischen zwei Studentinnen. Nachdem Uta in Zeile 121 ansetzt, um ihrer Freundin Lena einen Ratschlag zu geben, nimmt diese ihr die Äußerung quasi aus dem Mund, um den von Ute initiierten Handlungsvorschlag eigenmächtig fortzusetzen:

(5) BEZIEHUNGSKNATSCH (NRW 2011)

121 Uta: ich DENke echt eh?

122 d_das w_wäre das BESte,

123 Len: ${ }^{\circ} \mathrm{h}$ wenn ich mich bei IHM eh?

15 Von 16 kollaborativ erzeugten wenn-Konstruktionen sind 15 syntaktisch integriert, wobei in 13 Fällen die zweite Sprecherin die vom ersten Sprecher initiierte wenn-Konstruktion komplettiert (11 mal eingeleitet durch ein resumptives dann, wodurch die Anknüpfung stärker kontextualisiert wird). In einem Fall folgt einer initialen Protasis des ersten Sprechers (Kaj) eine durch die zweite Sprecherin (Uli) initiierte Fragehandlung:

239 Kaj: wenn_s halt (.) NET [ klappt-]

240 Uli:

ver ] gOht dir dann d_LUSCHT dran(. . .)?

Hier wird die vorausgehende wenn-Äußerung von der 2. Sprecherin retraktiv zur Projektorkonstruktion umfunktionalisiert. 
Uta: JA:hh.

Lena unterbricht mit ihrer Redezugübernahme (Z. 123) Utas Äußerungsproduktion, wobei sie deren vorausgehendes Syntagma „ich DENke echt eh? das w_wäre das BESte,“ rückwirkend als Anker (als Apodosis) für ihren folgenden wenn-Teilsatz „oh wenn ich mich bei IHM eh? hº e_entSCHULdige.“ (Z. 123f.) benutzt.

Dieser Ausschnitt verweist auf die „social-cognitive skills“ (Tomasello 2005: 19) der Interagierenden, die es ihnen ermöglichen, ,joint attentional frames“ aufzubauen und die kommunikativen Absichten des Gegenübers zu inferieren. ${ }^{16}$ Ono \& Thompson (1995: 232) argumentieren in Zusammenhang mit kollaborativ erzeugten grammatischen Schemata:

The schema instantiations which are started by one speaker and completed by another show that each speaker construes his/her contribution to be (part of) an instantiation of the same schema, and suggest that syntax cannot be thought of except as drawing upon cognitive and social resources.

(Ono \& Thompson 1995: 232)

Syntaktische Ko-Konstruktionen stellen keineswegs vereinzelte Ausnahmeerscheinungen dar, sondern sie repräsentieren kommunikative Praktiken, die Sprecherinnen und Sprecher für unterschiedliche kommunikative Zwecke einsetzen (Günthner 2015a, 2015b, 2020; Hilpert 2015). Während im vorliegenden Ausschnitt (5) BEZIEHUNGSKNATSCH die zweite Sprecherin den vorausgehenden Satz der ersten Sprecherin als Apodosis für ihre mit wenn-eingeleitete Protasis umfunktionalisiert, findet sich im folgenden Ausschnitt (einem Familientischgespräch) der umgekehrte Fall. Hier setzt die zweite Sprecherin (Ira) die von der ersten Sprecherin (Eva) initiierte wenn-Konstruktion „wenn du genÜgend vitaMIne (.) isst, (EIsen und_so,) auch HÜL(.)senfrüchte,“ (Z. 179-181) fort, indem sie die syntaktische Gestalt mit der Konsequenz „dann brauchste keine TIER[produkte.]“ (Z. 182) komplettiert:

(6) VEGANER (KÖLN 2001)

177 Eva: also da FEHLT ein_m echt [nIchts.]

$178 \mathrm{Kla}: \quad[\mathrm{hm}$.

179 Eva: wenn du genÜgend vitaMIne (.) isst,

16 Vgl. hierzu auch Du Bois’ (2014) Konzept der „dialogic syntax“, Linells (2009) Ansatz der „dialogicity“, Günthner, Imo \& Bücker (2014) zu Grammatik und Dialogizität sowie Hilpert (2015) zur „kollaborativen Insubordination“. 
180

181

182

183
(EIsen und_so, )

auch HÜL(. )senfrüchte,

Ira: dann BRAUCHste keine tIer[produkte.]

Kla:

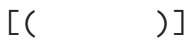

„Kollaborative Komplettierungen“ (Günthner 2015a, 2015b), die aus von verschiedenen Sprecherinnen und Sprechern erzeugten Komponenten bestehen, finden sich in Zusammenhang mit (integrierten) wenn-Konstruktionen immer wieder: Ein Sprecher beginnt mit der Initiierung der syntaktischen Gestalt und eine zweite Sprecherin nistet sich in die begonnene grammatische Struktur ein und führt diese zu Ende, indem sie die noch ausstehenden, projizierten Elemente beisteuert. Inwiefern Konstruktionsschemata „well entrenched“ (Langacker 1987: 59) sind, wird also u. a. daran ersichtlich, dass sie von mehreren Sprecherinnen und Sprechern gemeinsam im „konversationellen Duett“ produziert werden. Anhand kollaborativ erzeugter Gestalten wird somit erkenntlich, an welchen kognitiven Kategorien sich Sprecherinnen und Sprecher in der kommunikativen Realität tatsächlich orientieren und welche Konsequenzen die entsprechende Reaktivierung grammatischer Schemata für den interaktionalen Prozess hat. $^{17}$

\subsection{Jenseits biklausaler Satzmuster: Wenn-Konstruktionen, die längere Diskurseinheiten umfassen}

Im alltäglichen Gebrauch lassen sich - wie die Daten verdeutlichen - weder die wenn-Teile noch die Folgeeinheiten auf einen Satz bzw. Teilsatz begrenzen. Solche mehrere Diskurssegmente umfassenden Einheiten weichen erheblich vom scheinbar „prototypischen“, aus zwei Teilsätzen bestehenden Satzmuster der wenn-Konstruktion ab.

\section{a. Inkrementell expandierte wenn-Einheiten, die mehrere TCUs umfassen}

Sprecherinnen und Sprecher setzen oftmals wenn-Teile ein, die mehrere Teilsätze umfassen und sich über zahlreiche TCUs erstrecken. Jan und seine Nichte Mia unterhalten sich über den frühen Wintereinbruch, als Jan Mia rät, nicht mit dem

17 Hierzu auch Fischer (2015: 583).

18 Vgl. auch Günthner (2020). 
Rad zur Schule zu fahren. Jans wenn-Einheit (Z. $76 \mathrm{ff}$.) umfasst hierbei mehrere TCUs und beinhaltet u. a. dass-Sätze, Hauptsätze und Listenbildungen:

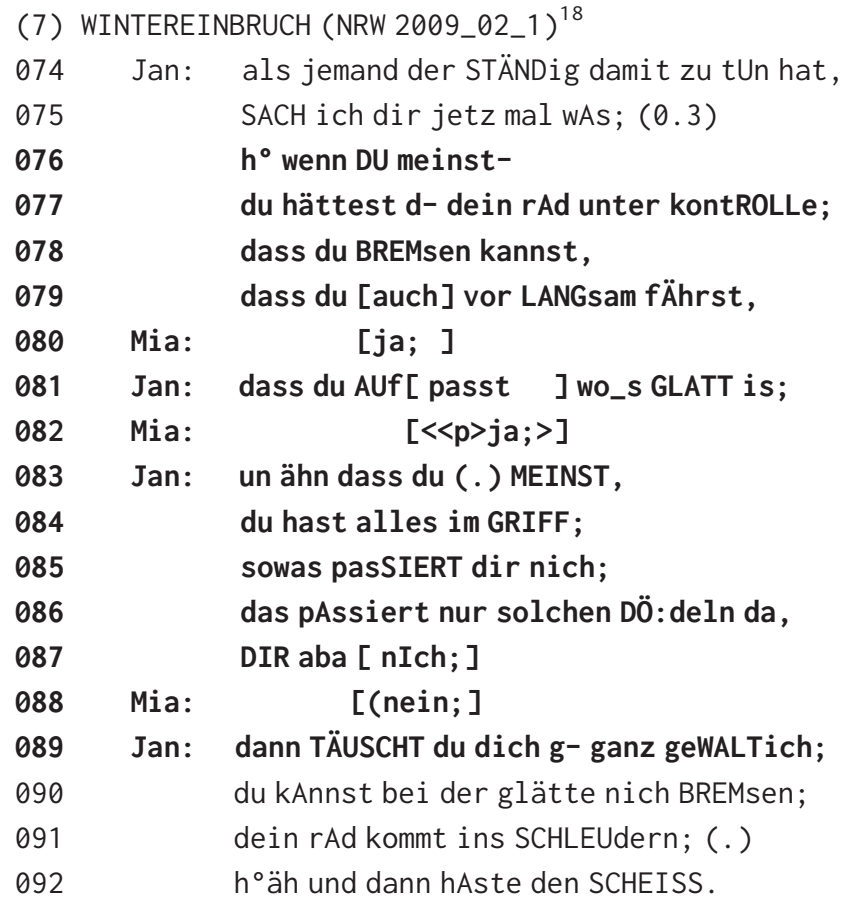

Auch wenn das von Jan in Zeile 076-077 produzierte wenn-Syntagma „ $\mathrm{h}^{\circ}$ wenn DU meinst- du hättest d- dein rAd unter kontROLLe;“ eine folgende Konsequenz projiziert, so veranschaulicht dieser Ausschnitt, dass das erwartbare Folgesegment keineswegs sofort erfolgen muss: Eine wenn-Einheit kann sich - wie im vorliegenden Fall - durchaus aus einer längeren, mehrere Teilsätze umfassenden Sequenz zusammensetzen. So wird die durch den Subjunktor wenn eingeleitete Protasis über mehrere Intonationseinheiten und syntaktische Konstruktionen hinweg inkrementell expandiert, so dass die projizierte Apodosis erst in Zeile 089 realisiert wird. Zugleich veranschaulicht das vorliegende Gesprächssegment ein weiteres Merkmal gesprochener Sprache: Syntaktische Strukturen bleiben nach ihrer Produktion für eine gewisse Zeit noch kognitiv verfügbar und können selbst über mehrere TCUs und Syntagmen hinweg für Folgeäußerungen aktiviert bleiben. Dennoch haben syntaktische Latenzen - wie alles in der gesprochenen Sprache - eine

18 Vgl. auch Günthner (2020). 
Zeitstruktur (Auer 2007; Deppermann \& Günthner 2015); d. h. sie verlieren im Prozess des Interaktionsverlaufs ihre Wirksamkeit. Aus diesem Grund verwenden Sprecherinnen und Spreher oftmals Strategien (wie Wiederholungen, Parallelismen, bestimmte Gesprächspartikeln etc.), die die entsprechende Anknüpfung kontextualisieren. Dies ist auch im vorliegenden Ausschnitt der Fall: Der in Zeile 076 initiierte wenn-Teil, dessen Projektion einer Folgekonsequenz über mehrere TCUs aktiviert bleibt, wird in Zeile 089 mittels des resumptiven dann re-kontextualisiert, sodass die komplexe, mehrere Diskurseinheiten umfassende syntaktische Formation kognitiv (re)aktiviert bleibt.

\section{b. Wenn-Teile, die von einer längeren Diskurseinheit gefolgt werden}

Nicht nur wenn-Teile werden oftmals inkrementell ausgeweitet und erstrecken sich über längere Sequenzeinheiten, sondern auch die Apodosis kann mehrere Teilsätze und Einheiten umfassen, wobei deren Ende oftmals nicht klar zu bestimmen ist (Günthner 1999; 2012, 2020; Wegner 2010). ${ }^{19}$ Im nächsten Ausschnitt reden Urs und Gabi über Urs' Studiensituation und dessen schlechte Erfahrungen mit der Studienberatung:

(8) STUDIUMSPLÄNE (BODENSEE 1998)

031 Urs: weiß ich ECHT net,

032 wie des GEHen soll,

033 aber (--) äh: vielLEICHT,

034 wenn ich jetzt nochmal zur STUdienberatung geh,

035 SICHerlich eh (.),

036 wär dann wieder dieser angnervte TYP do: $h$,

037 wo [mir] Immer $h^{\circ}$ WEIßmache will,

038 Gab: [hm ]

039 Urs: es_es gäb so die kombinatTION - (.)

040 die sei s0 net MöGlich; (. )

041 HIER anner Uni;

$042 \quad$ obwohl des gar net STIMMT.

043 i_im verZEICHnis der stUdiengänge,

044 stEhts nämlich sehr WOHL [drin.]

19 Vgl. Wegner (2010: 13), in dessen Daten 20\% der wenn-Konstruktionen eine komplexe Konsequenzkomponente aufweisen. 15\% seiner wenn-Teile umfassen mehrere TCUs und in $7 \%$ der in seinen Daten auftretenden wenn-Konstruktionen bilden sowohl Protasis als auch Apodosis komplexe Diskurssegmente. 
Bereits das adversative „aber“ in Kombination mit der Zögerungspartikel „äh:“ und dem mitigierenden „vielLEICHT“ (Z. 033) projizieren eine folgende Kontrastposition. Diese setzt mit dem wenn-Teilsatz ein: „wenn ich jetzt nochmal zur STUdienberatung geh,“ (Z. 034). Das mit steigender Endkontur realisierte wennSyntagma macht eine Fortsetzung der Ausführung erwartbar. Doch was folgt, ist keineswegs der prototypische Matrixsatz; vielmehr produziert der Sprecher eine (syntaktisch nicht-integrierte) komplexe Sequenz, die mehrere Teilsätze (durch wo eingeleiteter Relativsatz, indirekte Redewiedergabe, durch obwohl eingeleiteter Konzessivsatz etc.) enthält und sich über zahlreiche TCUs erstreckt: „,sicherlich eh (.),wär dann wieder dieser angnervte TYP do:h, wo [mir] Immer $\mathrm{h}^{\circ}$ WEIßmache will, es_es gäb so die kombinaTION - (.) die sei sO net MÖGlich; (.) HIER anner Uni; obwohl des gar net STIMMT. i_im verZEICHnis der stUdiengänge,stEhts nämlich sehr WOHL [drin.] (. . .)“ (Z. 035-044).

Auch hier fungiert der initial positionierte wenn-Satz im Sinne einer Projektorkonstruktion, die den Hintergrund für die projizierte Folgeargumentation liefert. Ferner illustriert diese wenn-Konstruktion einmal mehr, dass obgleich initiale wenn-Teilsätze eine Fortsetzung erwartbar machen - grammatische Projektionen keineswegs mit Determinationen gleichzusetzen sind. Die Form und der Inhalt des auf den wenn-Teil folgenden Syntagmas wird emergent ausgehandelt:

The notion of emergence constitutes a break with standard ideas about grammar that envisage it as a fixed synchronic system. It relativizes structure to speakers' actual experience with language, and sees structure as an on-going response to the pressure of discourse rather than as a pre-existent matrix. It follows that accounts of grammatical (and phonological) structure must take note of how frequency and repetition affect and, ultimately, bring about form in language.

(Bybee \& Hopper 2001: 3)

Solche sich über längere Diskurssequenzen hinweg erstreckende Konstruktionen veranschaulichen, wie in der kommunikativen Praxis interaktionale Faktoren mit kognitiven Aspekten zusammenwirken: Die interaktive Aushandlung grammatischer Formationen erweist sich als eng vernetzt mit Gedächtnisleistungen, die die betreffenden grammatischen Gestalten über größere Latenzen hinweg aktiviert halten. ${ }^{20}$

20 S. Fischer (2015) zu interaktionalen und kognitiven Aspekten in Zusammenhang mit grammatischen Konstruktionen. 


\subsection{Fragmentarisch verdichtete wenn-Konstruktionen}

Sprecherinnen und Sprecher setzen in Alltagsinteraktionen jedoch nicht nur sich über längere Sequenzeinheiten erstreckende, emergent produzierte Protasis- und Konsequenzteile ein, sondern sie produzieren auch extrem verdichtete wennSegmente. Diese Verdichtung ist so weit fortgeschritten, dass die Subjunktion wenn durchaus als alleinstehendes Element verwendet werden kann (Auer 2002; Günthner 2012).

Der folgende Ausschnitt entstammt wiederum der bereits oben erwähnten Reality TV-Show. Die Container-Bewohnerinnen und -Bewohner unterhalten sich darüber, welche Lebensmittel sie in den Garten zum Essen mitnehmen wollen, da ihnen dort in wenigen Minuten die Entscheidung, wer von ihnen in der Show bleiben darf und wer gehen muss, verkündet werden soll:

(9) BROT MITNEHMEN (REALITY-TV-SHOW: bb \# 198)

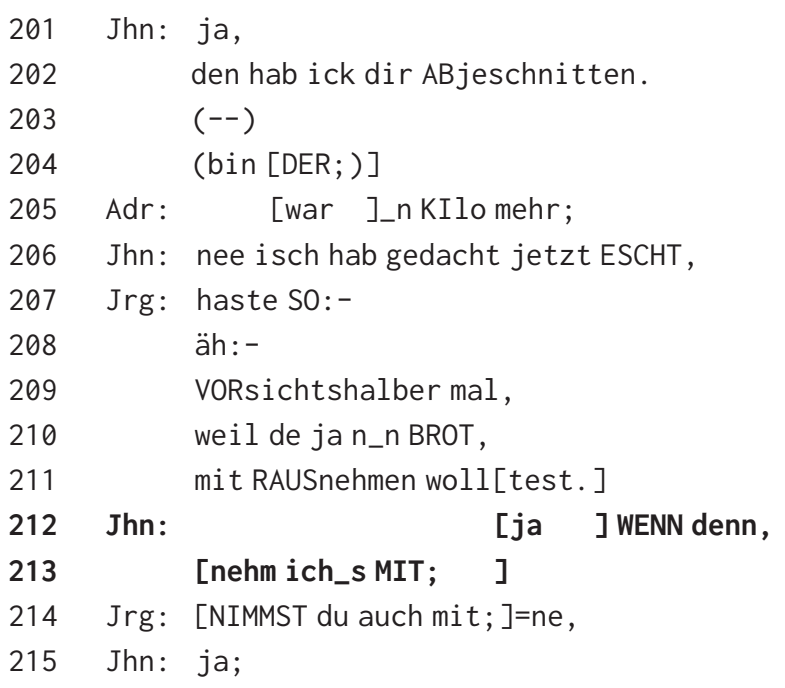

In Zeile 212 initiiert John seinen Redebeitrag mit der Zustimmungspartikel ,ja“ gefolgt von dem mit Hauptakzent markierten Subjunktor „WENN“. Statt der Produktion eines wenn-Teilsatzes setzt John unmittelbar nach dem Subjunktor seine Äußerung mit dem resumptiven „denn“ fort und markiert so den Beginn der Konsequenz: „denn nehm ich_s MIT.“ (Z. 212-213). Letztere erfolgt in Überlappung mit Jürgens kollaborativem Anschluss „,[NIMMST du auch mit;] = ne,“ (Z. 214), der wiederum Johns wenn-Fragment als Anker seiner wenn-dann-Komplettierung nimmt. 
Die Protasis ist hier also auf ein (ja) wenn-Fragment reduziert, das aufgrund seiner sequenziellen Einbettung allerdings problemlos interpretierbar ist. Die vom zweiten Sprecher (Jürgen) in Überlappung produzierte Fortsetzung macht deutlich, dass selbst fragmentarische wenn-Konstruktionen grammatische Schemata bilden, die Bestandteil des Wissenshaushalts der Interagierenden sind und als solche auch im Prozess der Interaktion interpretiert und behandelt werden.

Der folgende Ausschnitt entstammt einem Familientischgespräch: Die Mutter (Mut), der Sohn Andreas (And) und dessen Frau Carola (Car) unterhalten sich über ein extrem großes Baby in ihrer Nachbarschaft:

(10) GROBES BABY (SCHWABEN 17, DAT: 11.25)

324 Mut: ja du musch ja RECHne,

325 des kind wA: $r$ ja scho GROß- (.)

326 wo_s uff d_WELT komme isch;

327 Car: wenn de STILLSCH,

328 na kannsch NIE überernähre;

329 WENN, (.)

330

331

332 And: [nei ] die meinet da des Breile und so: ;

Nachdem die Mutter ausführt, dass das besagte Baby bereits bei der Geburt „scho GROß-“ war (Z. 325), setzt Carola mit einem wenn-Satz ein und betont, dass Kinder, die gestillt werden, „NIE“ überernährt sein können (Z. 328). Nach Abschluss dieses wenn-dann-Gefüges setzt sie ihren Redezug mit einem akzentuierten „WENN“ fort; doch statt einen wenn-Teilsatz zu formulieren, ist die Protasis auf das prosodisch markierte „WENN“ verdichtet. Das resumptive „dann“ in Zeile 330 markiert daraufhin den Beginn der Konsequenz.

Während in den Ausschnitten (9) BROT MITNEHMEN und (10) GROßES BABY Ellipseninterpretationen problemlos möglich sind, ist diese im folgenden Gesprächsausschnitt bereits schwieriger. Greta (Gre) und Hans (Han) besprechen, inwiefern sie einen alten Tisch neu herrichten können. Nachdem Greta den Vorschlag gemacht hat, dass man notfalls „einfach schnell mal vier Füße an den Tisch bastelt“, äußert Hans seine Bedenken, dass dies doch mehr Arbeit sei, als sich Greta vorstellt:

21 „Schoppe“ ist dialektal für „Babyfläschchen“. 


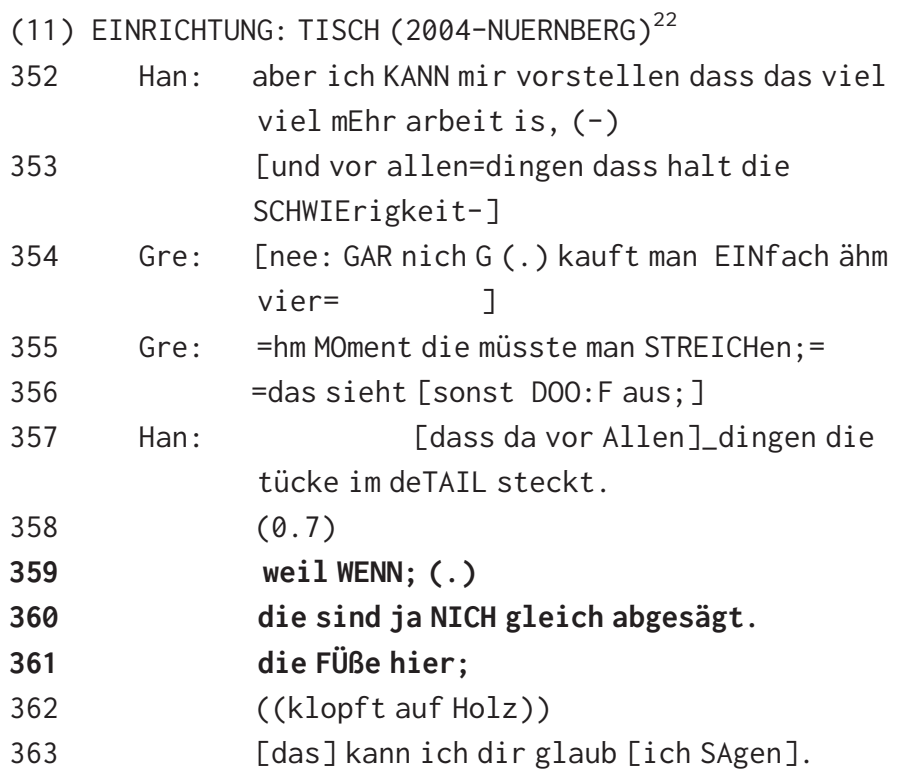

Hans thematisiert in Zeile 352-353 seine Bedenken bzgl. des schnellen „Bastelns“ von vier Füßen: „dass da vor Allen_dingen die tücke im deTAIL steckt.“ (Z. 357). In Zeile 359 setzt er mit einer Begründung seiner Einschätzung durch weil ein; ${ }^{23}$ doch formuliert er statt eines ausformulierten wenn-Teilsatzes nur das Protasisfragment („wenn“). Die Folgeeinheit, die eine eigenständige Intonationseinheit repräsentiert (Z. 360), liefert schließlich die inhaltliche Begründung für seine Skepsis („die sind ja NICH gleich abgesägt“), die allerdings nicht als Apodosis zur fragmentarischen Protasis fungiert. ${ }^{24}$

Fragmentarische wenn-Syntagmen verdeutlichen einmal mehr, dass auch solche von den Normen der Standardsprache abweichende Formate Teil unseres sprachlichen Wissenshaushalts darstellen und als Ressourcen zur Durchführung spezifischer kommunikativer Aufgaben in Alltagsinteraktionen verwendet werden (Günthner 2011a, 2011b). Analysen und Theoriebildungen, die auf Sprache im Medium der Schrift reduziert sind, ignorieren somit einen zentralen Bereich sprachlicher Produktivität und verlieren die Vielfalt und Flexibilität des Sprachgebrauchs aus den Augen (vgl. Ágel 2003), der in diesem Zusammenhang vom „Skriptizismus“ der Linguistik redet, und Linell

22 Hierzu auch Günthner (2012).

23 Die vorliegende Verwendung von weil fungiert als Diskursmarker (Günthner 1999).

24 Siehe Auer (2002) zur Verdichtung der konditionalen Hypotaxe im gesprochenen Deutsch. 
(2005), der den „written language bias“ sprachwissenschaftlicher Forschung kritisiert).

\subsection{Alleinstehende wenn-Konstruktionen}

Bislang habe ich argumentiert, dass initiale wenn-Teilsätze (ja selbst wenn-Fragmente) prosodisch, syntaktisch, semantisch und pragmatisch unvollständige Einheiten repräsentieren, die eine Fortsetzung auf Seiten der Sprecherin / des Sprechers erwartbar machen. In Alltagsinteraktionen trifft man allerdings immer wieder auf wenn-Einheiten, denen weder ein Matrixsatz folgt, noch vorausgeht (Günthner 2012, 2017, 2020). Solche „alleinstehenden wenn-Sätze“ sind keineswegs unvollständige Einheiten, sondern sie bilden eigenständige kommunikative Projekte.

„Unabhängige wenn-Sätze“ wurden von zahlreichen Grammatikern immer wieder diskutiert: Im Allgemeinen werden solche „unabhängigen Verbendsätze“ bzw. „alleinstehenden untergeordnete Sätze“ (Brugmann 1918; Weuster 1983; Altmann 1987; Oppenrieder 1989; Scholz 1991) mit spezifischen Satztypen assoziiert, wie mit „Optativen“, die Wünsche (,wenn ich doch bloß Millionär wäre!“) oder Bitten (,wenn ich um eine Antwort bitten dürfte“) ausdrücken. Im Folgenden werde ich verdeutlichen, dass die Reichweite alleinstehender wennSätze weit über die traditionell beschriebenen Funktionen hinausgeht. ${ }^{25}$

\section{a. Optative}

Wunschsätze in der Form eines „isolierten wenn-Satzes“ werden in zahlreichen Grammatiken erwähnt und als verselbstständigte Form ehemaliger Nebensätze eingeordnet (hierzu auch Riesel 1964: 166; Buscha 1976: 275; Hoffmann 2013: 544; Zifonun, Strecker \& Hoffmann 1997: 668). Wie der folgende Gesprächsausschnitt zeigt, ist die Abgrenzung zwischen Optativsätzen und Exklamationsäußerungen oftmals fließend, zumal die (irrealen) Wünsche meist als Ausrufe produziert werden, bei denen „eine spontane Empfindung von Ungewöhnlichkeit eines gegebenen Sachverhalts oder einer Sachverhaltsdimension“ zum Ausdruck kommt (Hoffmann 2013: 542; Günthner 2017: 100).

Der folgende Ausschnitt entstammt einem onkologischen Aufklärungsgespräch zwischen der Ärztin (AW), der Patientin (PW) und deren Lebensgefährten (LM). Nachdem die Ärztin die Patientin über deren Brustkrebsdiagnose

25 Hierzu auch Günthner (2017, 2020). 
aufgeklärt hat, besprechen die Anwesenden mögliche Therapiemaßnahmen. Hierbei kommt die Frage nach dem Grund für die Erkrankung auf:

(12) BRUSTKREBS (GESPRÄCH 069) ${ }^{26}$

176 AW: weil das wichtigste für sIe dass sie geSUND werden,

177 ist dass der tumor RAUS aus dem körper kommt.

$178 \quad(1.5)$

179 PW: $<<p>$ ja.>

180 LM: ${ }^{\circ} \mathrm{h}$ von WAS kommen diese-

181 AW: wenn man wüsSte wovon [brustkrebs] kommt;

182 LM: [art, ]

183 LM: ja meine liebe es gibt es gibt milliARden sogar,

184 bis JETZT;

185 schon seit hundertmillionen jahre invesTIERT man,

186 und KEIner weiß bescheid,

$187 \quad$ AW: $[<<p>j a ;>]$

188 LM: [KEINen ] millimeter weiter;

AWs redezugkonstituierende Äußerung „wenn man WÜSSte wovon [brustkrebs] kommt, “ in Zeile 181 weist typische Merkmale eines abhängigen Teilsatzes auf: Sie setzt mit der Subjunktion wenn ein und zeigt Verbendstellung (,WÜSSte“). Jedoch folgt kein Matrixsatz, sondern das wenn-Syntagma repräsentiert eine eigenständige Sprechhandlung: einen exklamativ ausgedrückten Wunsch. Dies wird durch die Reaktion des Rezipienten LM bestätigt, der im Anschluss an AWs wenn-Äußerung seine Lebenspartnerin darüber belehrt, dass „KEIner“ über die Ursachen von Brustkrebs „bescheid“ weiß (Z. 186). Bezeichnend ist ferner, dass der vorliegende, gesprochensprachliche wenn-Wunschsatz ,wenn man WÜSSte wovon [brustkrebs] kommt,“ (Z. 181) keine der laut Forschungsliteratur für Wunsch- und Exklamativsätze erforderlichen Partikeln doch, nur, bloß, ja wenigstens enthält. ${ }^{27}$

Alleinstehende wenn-Teilsätze, die syntaktisch subordiniert sind, aber eigenständige prosodische und handlungsbezogene Aktivitäten repräsentieren, entsprechen den von Evans (2007: 367) als „insubordination“ bezeichneten

26 Dieser Ausschnitt entstammt dem von Prof. Dr. med. Martin Bentz, Prof. Dr. med. Thomas Rüdiger und Prof. Dr. Wolfgang Imo geleiteten Forschungsprojekt „Von der Pathologie zum Patienten: Optimierung von Wissenstransfer und Verstehenssicherung in der Onkologie zur Verbesserung der Patientensicherheit“ (gefördert von der Deutschen Krebshilfe; Projektnummer 111172).

27 Hierzu auch Günthner (2017: 102). 
Phänomenen: „the conventionalized main clause use of what, on prima facie grounds, appear to be formally insubordinate clauses" (Hervorhebung im Original; S.G.). Auffallend bei diesen alleinstehenden wenn-Syntagmen ist somit der Widerspruch zwischen der Form (d. h. den Subordinationsindikatoren), die grammatische Unselbstständigkeit markiert, und der mit dieser Form ausgeführten eigenständigen kommunikativen Handlung (Günthner 2015a, 2017, 2020; Hilpert 2015).

Wie die vorliegenden Daten zeigen, verwenden Sprecherinnen und Sprecher alleinstehende, nicht-projizierende wenn-Konstruktionen auch in Zusammenhang mit der Durchführung weiterer Aktivitäten (jenseits von Optativen); u. a. zur Kontextualisierung von Drohungen/Warnungen sowie zum exklamativen Ausdruck von Bewertungen.

\section{b. Drohungen/Warnungen}

Der folgende Ausschnitt entstammt einer Reality-Show im Fernsehen. Sabrina (Sab) rekonstruiert eine Episode aus ihrer Kindheit, als sie sich fast einen Finger abgeschnitten hat, doch dies - aus Angst vor ihrer strengen Mutter - verheimlichen wollte. Am folgenden Tag ist die Wunde aufgeplatzt, so dass die Mutter den Unfall bemerkt hat und „stinkesauer“ auf Sabrina war:

(13) STRENGE MUTTER (REALITY-TV-SHOW: bb \# 17$)^{28}$

228 Sab: <all> un_am nächsten TACH hat_sie irgendwie,>

229 die (.) EINkaufstüte (so),

230 die hat das nIch geMERKT,

$231 \quad<<a l l>$ und dann hat- $(-)$

232 sie dann das ge[SEHN, ] ne>

233 Jür: [ja. ]

234 Sab: und dann HAT_se dann-

235 (weißt halt )(.) da war der ganze verband alles Voll [ BLU:T.]

236 Jür: $[<<p>h m]$

$237 \quad(0.5)$

238 Sab: und dann HATse,

239 <<flüsternd> wenn du DAS noch mal mAchst.>

$240 \quad(--)$

$241<<p>$ meine mutter war STRENG.>

242 Jür: ja aber das ist doch n_!UN!fall.>

28 Vgl. auch Günthner (2020). 
Die Drohung der Mutter wird hier in direkter Rede (mit flüsternder, affekt-geladener Stimme) in Form einer alleinstehenden wenn-Konstruktion rekonstruiert: „<<flüsternd> wenn du DAS noch mal mAchst. >“ (Z. 239). Die in das wenn-Syntagma verpackte Erwähnung der Wiederholung „noch mal“ in Kombination mit Kontextualisierungsverfahren wie der markierten prosodischen Gestaltung und dem Hinweis auf eine zukünftige Handlung der Rezipientin etc. scheinen für die Tochter ausreichend, um die Ernsthaftigkeit der mütterlichen Drohgebärde in Gestalt einer Aposiopese (Imo 2014: 163f.; Auer 2007: 105) $\mathrm{zu}$ verstehen. Drohungen in Form von wenn-Konstruktionen - kombiniert mit spezifischen prosodischen und handlungsrelevanten Elementen - sind bereits derart routinisiert, dass sie keine explizite Konsequenzformulierung benötigen. ${ }^{29}$

\section{c. Exklamationen und der Ausdruck von Bewertungen bzw. „stances“}

Nach Evans (2007: 392) stellen vor allem Realisierungen von „interpersonal coercion“ (Drohungen, Warnungen, Wünsche etc.) typische Aktivitäten dar, die in Zusammenhang mit insubordinierten Konstruktionen auftreten. Oftmals werden alleinstehende wenn-Konstruktionen auch - wie im folgenden Beispiel (14) - zum Ausdruck von Bewertungen, Nichtübereinstimmungen und affektgeladenen Exklamationen verwendet (Günthner 2017).

Der folgende Ausschnitt entstammt ebenfalls der oben zitierten TV-RealityShow. Die Bewohnerinnen und Bewohner der Container-WG streiten darüber, dass im Kühlschrank stets neue Joghurt- bzw. Frischkäsetöpfchen verwendet werden, statt die bereits geöffneten aufzubrauchen. Nachdem Steffi (Ste) ihre Mitbewohnerin Anne (Ann) beschuldigt, dass diese vor kurzem ,alle“ im Kühlschrank vorfindlichen Packungen ,aufgemacht“ hat, obwohl bereits geöffnete im Kühlschrank lagen, streitet Letztere dies ab und wiederholt daraufhin Steffis Vorwurf, indem sie deren Anschuldigung (deiktisch angepasst) reproduziert: „<<t> ich hab die Alle AUFgemacht. >“ (Z. 335). Das tiefe Tonhöhenregister, die Akzenttonhöhenbewegung auf „AUF“ und die tief fallende Tonhöhenbewegung am Ende der Kontur markieren eine indignierte Stimme:

29 So argumentiert auch Evans (2007: 390), dass im Fall stark konventionalisierter insubordinierter „if-clauses“ die Rekonstruktion eines scheinbar getilgten Hauptsatzes kaum noch möglich ist. Hierzu Günthner (2017: 104-105). 
(14) WENN ICH DAS SCHON HÖR (REALITY-TV-SERIE \#2) ${ }^{28}$

$\begin{array}{lrl}331 & \text { Ann: } & {[<<>\text { un }]} \\ 332 & & \text { das war sOnntag vor EIner wOche. }> \\ 333 & & (3.8) \\ 334 & & <<\mathrm{t}, \mathrm{p}>\mathrm{p}-\mathrm{hm} \text { ECHT ey; }> \\ 335 & & <<\mathrm{t}>\text { ich hab die Alle AUFgemacht. }>(--) \\ 336 & & <<\mathrm{t}>\text { wenn ich dAs schon HÖR; }> \\ 337 & \text { Ste: } & <<\mathrm{f}>\text { nein nich ALle? }> \\ 338 & & {[\text { sondern wenn es jemand; }>]} \\ 339 & \text { Ann: } & {[<<\mathrm{f}>\text { hAst du aber geSACHT; }]}\end{array}$

Im Anschluss an die Vorwurfsrekonstruktion „«<t> ich hab die Alle AUFgemacht. > (Z. 335) setzt Anne nach einer kurzen Pause ihren Redezug mit einer alleinstehenden wenn-Konstruktion fort „ $<<\mathrm{t}>$ wenn ich dAs schon HÖR; $>$ “ (Z. 336). Bei dieser wenn-Äußerung handelt es sich um eine affektgeladene Exklamation, mit der Anne ihre Entrüstung zum Ausdruck bringt. Als unmittelbare Reaktion auf diese Indignation streitet Steffi ab, dass sie Anne beschuldigt habe, „ALLe“ Packungen aufgemacht zu haben (Z. 337); vielmehr ging es ihr lediglich darum, dass sie es nicht gut finde, wenn jemand eine neue Packung aufmache, nur weil er/sie nicht aus einer angebrochenen Packung essen wolle.

Auch wenn die vorliegende wenn-Äußerung , $<<t>$ wenn ich dAs schon HÖR; $>$ (Z. 336) syntaktisch „nicht vollständig“ ist, so wird dieses formelhafte wenn-Syntagma von den Beteiligten als eigenständige kommunikative Handlung interpretiert: Im Anschluss übernimmt Steffi das Rederecht und produziert ihre Rechtfertigung. Die prosodische Realisierung alleinstehender wenn-Konstruktionen untermauert auch hier deren kommunikative Selbstständigkeit: Der wenn-Satz wird als eigenständige Einheit mit meist fallender Endkontur realisiert.

Die Beobachtungen zur prosodischen Gestaltung alleinstehender wennKonstruktionen verfestigen die Annahme eines produktiven Zusammenhangs zwischen prosodischer Realisierung und kommunikativer Funktion grammatischer Konstruktionen im Gespräch: Die prosodische Gestaltung fungiert als Kontextualisierungshinweis und liefert eine wichtige semiotische Grundlage für die Interpretation grammatischer Gestalten (Gumperz 1982). Alleinstehende wenn-Konstruktionen, die Wünsche, Drohungen, Warnungen und affektiv aufgeladene Einstellungen übermitteln, scheinen im Deutschen bereits derart „entrenched“ (Langacker 1987) zu sein, dass man sie als „grammatikalisierte Muster“ bzw. Konstruktionen bezeichnen kann. Zugleich widersprechen sie den klassi-

30 Vgl. Günthner (2017: 106). 
schen Annahmen der Unidirektionalität in Hinblick auf syntaktische Komplexitätsentwicklung (Hilpert 2015: 28). So geht die Grammatikalisierungstheorie davon aus, dass syntaktisch komplexe Satzgefüge durch Kombination und Integration aus ursprünglich unabhängigen Satzstrukturen entstehen. ${ }^{31}$ Doch scheint hier die umgekehrte Richtung vorzuliegen: Alleinstehende subordinierte Konstruktionen entwickeln sich - so Evans (2007) - aus komplexen Strukturen und verselbständigen sich im Laufe der Zeit durch Tilgung des Matrixsatzes. ${ }^{32}$

Allerdings entstehen keineswegs aus allen komplexen Satzgefügen (subordinierten Teilsätzen + Matrixsätze) Formen der Insubordinationen. Gerade in Bezug auf wenn-Konstruktionen wird deutlich, dass alleinstehende wennSätze spezifische interaktive Funktionen innehaben und somit aktivitätsgebunden eingesetzt werden - nämlich primär in Kontexten, die ein erhöhtes Maß an dialogischer Orientierung aufweisen (vgl. auch Evans 2007; Hilpert 2015: 29).

\section{Schlussfolgerungen}

Ein holistischer Ansatz zur Analyse grammatischer Phänomene (wie von Seiten der Konstruktionsgrammatik postuliert) erfordert es, Aspekte des situierten Gebrauchs als integralen Teil der Untersuchung einzubeziehen (Günthner \& Imo 2006; Deppermann 2011). Zugleich vermittelt eine solche holistische Perspektive ein sehr viel komplexeres Bild grammatischer Phänomene als traditionelle, an der normierten Schriftsprache orientierte Analysen abgeben. So weisen auch

31 Vgl. u. a. Givón (2009: 2), der die Entstehung syntaktisch komplexer Satzgebilde aus der Integration ursprünglich unabhängiger Strukturen als typischen Grammatikalisierungsprozess beschreibt. S. auch Hopper \& Traugott (1993/2003: 168 ff.). Vgl. auch Ágel \& Höllein (i.d.B.) zur Modellierung komplexer syntaktisch-semantischer Sprachzeichen.

32 Wenn jedoch Insubordination ein Gegenbeispiel für die Annahme der Unidirektionalität im Grammatikalisierungsprozess darstellt und insubordinierte Konstruktionen (wie die insubordinierte wenn-Konstruktion) sich aus einem komplexen Satzgefüge durch zunehmende Tilgung des Matrixsatzes entwickelt hat, so erstaunen zunächst einmal die Ergebnisse von Diessel (2004) zum kindlichen Spracherwerb: Diessel (2004: 160) zeigt, dass die unabhängige Verwendung subordinierter Adverbialsätze oftmals der abhängigen Verwendung dieser Teilsätze mit voran- bzw. nachgestelltem Matrixsatz vorausgeht. Hierzu auch Hilpert (2015: 26-29). Allerdings könnte dieser Befund auch damit zusammenhängen, dass Eltern gegenüber Kindern häufig alleinstehende wenn-Sätze bei Drohungen und Warnungen verwenden. Diesen Hinweis verdanke ich Verena Wecker. 
die vorliegenden Ergebnisse zu wenn-Konstruktionen im Alltagsgebrauch darauf hin, dass Sprecherinnen und Sprecher über eine Vielfalt an durchaus routinisierten Varianten von wenn-Konstruktionen verfügen, die als ,a continuum reflecting the syntatic modes which speakers of German tend to use in actually occurring speech“ (Köpcke \& Panther 1989: 709) interpretiert werden können und unterschiedliche Grade an syntaktischer (prosodischer, semantischer, sequenzieller und handlungsbezogener) Integration zwischen dem subordinierten wenn-Teil und der Folgeeinheit zeigen:

\begin{tabular}{|c|c|c|c|c|}
\hline \multicolumn{5}{|c|}{ wenn-Konstruktionen im Gebrauch } \\
\hline $\begin{array}{l}\text { syntaktische } \\
\text { Integration } \\
\qquad \mid \\
\text { enge syntakt. } \\
\text { Verknüpfung } \\
\text { der beiden } \\
\text { Einheiten (von } \\
\text { prosodisch } \\
\text { integriert zu } \\
\text { nicht integriert) }\end{array}$ & $\begin{array}{l}\text { Synta } \\
\text { Desin } \\
\text { synt. und prosod. } \\
\text { Nicht-Integration } \\
\text { (a) konvertibel } \\
\text { (b) nicht } \\
\text { konvertibel }\end{array}$ & $\begin{array}{l}\text { jenseits } \\
\text { biklausaler } \\
\text { Konstruktionen } \\
\text { (a) inkrementell } \\
\text { expandierende } \\
\text { wenn-Einheit } \\
\text { (b) Folgeeinheit } \\
\text { umfasst eine } \\
\text { längere Sequenz }\end{array}$ & $\begin{array}{l}\quad \mid \\
\text { auf wenn } \\
\text { verdichtete } \\
\text { Protasis }\end{array}$ & $\begin{array}{l}\text { alleinstehende } \\
\text { wenn-Einheiten } \\
\text { ohne folgenden } \\
\text { Matrixsatz }\end{array}$ \\
\hline
\end{tabular}

Abb. 1: wenn-Konstruktionen im Gebrauch.

Die in der mündlichen Interaktion auftretenden grammatischen Muster zeigen somit ein differenzierteres Bild von grammatischen Konstruktionen, als das statische Konzept einer 1:1-Form-Funktions-Paarung zulässt: Statt homogener, stabiler Form-Funktions-Paarungen liegen wenn-Formate vor, die sich als relativ „flexibel“ einsetzbar für vielfältige adaptive Verwendungen im Prozess kommunikativen Handelns erweisen (Deppermann 2011: 229; Hopper 2004, 2011; Günthner \& Hopper 2010). Zugleich werfen die Befunde die Frage nach der „Identität von Konstruktionen“ (Deppermann 2011: 229-230) bzw. der Konzeptualisierung der unterschiedlichen wenn-Formate auf. Inwiefern lassen sich die vorliegenden wenn-Varianten als „abstract prototypes“ (Ono \& Thompson 1995: 217) konzeptu- 
alisieren, an denen sich Sprecherinnen/Sprecher und Rezipientinnen/Rezipienten im interaktionalen Gebrauch orientieren und die sie flexibel und kontextkontingent einsetzen bzw. interpretieren? Oder lässt sich aus diesen Varianten eine grundlegende bzw. prototypische wenn-Konstruktion ermitteln und wenn ja, auf welcher Grundlage und mit welchem Granularitätsgrad? (Imo 2011; Deppermann 2011; Günthner 2015c)? In Anlehnung an Croft (2001) könnte man argumentieren, dass die vorliegenden routinisierten wenn-Formate Teil eines strukturierten Inventars bilden, das verwandte Konstruktionen über ein Netzwerk miteinander verknüpft. Jeder Konstruktionstyp würde hierbei - aufgrund seiner syntaktischen, lexikalischen, prosodischen und interaktional-sequenziellen Merkmale einen eigenen Knoten im Konstruktionsnetzwerk darstellen (Croft 2001: 25-27). Zugleich müsste jedoch die Dynamik und situierte Prozesshaftigkeit der wennRealisierungen erfasst werden, die den verschiedenen Varianten einen flexiblen, kontextkontingenten Gebrauch erlauben: Grammatische Konstruktionen repräsentieren nun mal „something that people do rather than something they have.“ (Langacker 2008: 216).

Ferner zeigt die Analyse, dass wenn-Konstruktionen im Alltagsgebrauch eng mit interaktionalen und kognitiven Prinzipien verwoben sind. Hierzu zählen die inhärente Dialogizität sprachlichen Handelns, die Prozessualität und Emergenz grammatischer Strukturbildung, die sequenzielle Organisation von sprachlichen Strukturen etc., wie auch geteilte Aufmerksamkeiten, wechselseitige Relevanzsetzungen sowie Gedächtnisleistungen, die grammatische Gestalten über längere Latenzen hinweg aktiviert bleiben lassen. Eine gebrauchsbasierte, an der interaktiven Realität ausgerichtete Perspektive auf Konstruktionen bzw. Schemata ermöglicht es, diese nicht länger auf eine Rekonstruktion ,in der Domäne der Kognition und entlang taxonomischer Hierarchien“ zu reduzieren, sondern die Domäne des situierten „Zeichenhandelns“ (Feilke \& Linke 2009: 5) und damit den Ort, wo Konstruktionen bzw. „Schemata gebrauchsbasiert geprägt werden“ (Bücker 2015: 448), in den Blick zu nehmen. Auf diese Weise erhält man Einblick in die Vorgänge der ,online'-Prozessierung von grammatischen Mustern.

\section{Literatur}

Altmann, Hans (1987): Zur Problematik der Konstitution von Satzmodi als Formtypen. In Jörg Meibauer (Hrsg.), Satzmodus zwischen Grammatik und Pragmatik, 22-56. Tübingen: Niemeyer.

Ágel, Vilmos (2003): Prinzipien der Grammatik. In Anja Lohenstein-Reichmann \& Oskar Reichmann (Hrsg.), Neue historische Grammatiken. Zum Stand der Grammatikschreibung 
historischer Sprachstufen des Deutschen und anderer Sprachen, 1-46. Tübingen: Niemeyer.

Ágel, Vilmos (2016): Obwohl (.) fährt der eigentlich auch am Sonntag? Der Verbzweit-Mythos. In Brigitte Handwerker, Rainer Bäuerle, \& Bernd Sieberg (Hrsg.), Gesprochene Fremdsprache Deutsch, 75-100. Baltmannsweiler: Schneider Verlag Hohengehren.

Ágel, Vilmos (2017): Grammatische Textanalyse. Textglieder, Satzglieder, Wortgruppenglieder. Berlin u. a.: De Gruyter.

Ágel Vilmos \& Dagobert Höllein (i. d. B.): Satzbaupläne als Zeichen: die semantischen Rollen des Deutschen in Theorie und Praxis.

Auer, Peter (1996): The pre-front field position in spoken German and its relevance as a grammaticalization position. Pragmatics 6 (3), 295-322.

Auer, Peter (2000): Pre- and post-positioning of wenn-clauses in spoken and written German. In Elizabeth Couper-Kuhlen \& Bernd Kortmann (Hrsg.), Cause, condition, concession, contrast: Cognitive and discourse perspectives, 173-204. Berlin: De Gruyter.

Auer, Peter (2002): Die Verdichtung der konditionalen Hypotaxe im gesprochenen Deutsch. In Vilmos Ágel \& Andreas Herzog (Hrsg.), Jahrbuch der ungarischen Germanistik 2002, 189-204. Budapest/Bonn: DAAD.

Auer, Peter (2007): Syntax als Prozess. In Heiko Hausendorf (Hrsg.), Gespräch als Prozess. Linguistische Aspekte der Zeitlichkeit verbaler Interaktion. 95-124. Tübingen: Narr.

Auer, Peter \& Stefan Pfänder (2011): Constructions: Emergent or emerging? In Peter Auer \& Stefan Pfänder (Hrsg.), Constructions: Emerging and emergent, 1-21. Berlin u. a.: De Gruyter.

Auer, Peter \& Jan Lindström (2015): Left/right asymmetries and the grammar of prevs. postpositioning in German and Swedish talk-in-interaction. InLiSt: Interaction and Linguistic Structures 56. http://www.inlist.uni-bayreuth.de/ (3. Juli 2018).

Binanzer, Anja, Jana Gamper \& Verena Wecker (i. d. B.): Einleitung.

Bittner, Andreas \& Klaus-Michael Köpcke (2007): Überlegungen zur Repräsentation grammatischen Wissens am Beispiel der Verbmorphologie des Deutschen. In Claudio Di Meola, Livio Gaeta, Antonie Hornung \& Lorenzo Rega (Hrsg.), Perspektiven Zwei. Akten der 2. Tagung ,Deutsche Sprachwissenschaft in Italien', Rom, 9.-11.2.2006, 3-15. Rom: Herder.

Bittner, Dagmar (i. d. B.): Strukturalistische vs. gebrauchsbasierte Modellierung des Erwerbs der definiten Artikel und D-Pronomen des Deutschen.

Brenning, Jana (2012): Speakers' orientation to the nucleus accent in syntactic coconstructions. In Pia Bergmann, Jana Brenning, Martin Pfeiffer \& Elisabeth Reber (Hrsg.), Towards an interactional grammar, 74-102. Berlin u. a.: De Gruyter.

Brugmann, Karl (1918): Verschiedenheit der Satzgestaltung nach Maßgabe der seelischen Grundfunktionen in den indogermanischen Sprachen. Leipzig: Teubner.

Bybee, Joan \& Paul Hopper (2001): Introduction to frequency and the emergence of linguistic structure. In Joan Bybee \& Paul Hopper (Hrsg.): Frequency and the emergence of linguistic structure, 1-24. Amsterdam: Benjamins.

Bücker, Jörg (2015): Schema - Muster - Konstruktion. In Christa Dürscheid \& Jan Georg Schneider (Hrsg.), Handbuch Satz, Äußerung, Schema, 445-463. Berlin u. a.: De Gruyter.

Bücker, Jörg, Susanne Günthner \& Wolfgang Imo (2015) (Hrsg.), Konstruktionsgrammatik V: Konstruktionen im Spannungsfeld von sequenziellen Mustern, kommunikativen Gattungen und Textsorten. Tübingen: Stauffenburg. 
Buscha, Annerose (1976): Isolierte Nebensätze im dialogischen Text. Deutsch als Fremdsprache 13, 274-279.

Comrie, Bernard (1986): Conditionals: A typology. In Elizabeth Traugott, Alice ter Meulen, Judy S. Reilly \& Charles A. Ferguson (Hrsg.), On conditionals, 77-99. Cambridge: Cambridge University Press.

Croft, William A. (2001): Radical construction grammar. Oxford: Oxford University Press.

Dausendschön-Gay, Ulrich, Elisabeth Gülich \& Ulrich Krafft (2015) (Hrsg.), Ko-Konstruktionen in der Interaktion. Die gemeinsame Arbeit an Äußerungen und anderen sozialen Ereignissen. Bielefeld: transcript.

Deppermann, Arnulf (2011): Konstruktionsgrammatik und Interaktionale Linguistik: Affinitäten, Komplementaritäten und Diskrepanzen. In Alexander Lasch \& Alexander Ziem (Hrsg.), Konstruktionsgrammatik III - Aktuelle Fragen und Lösungsansätze, 205-238. Tübingen: Stauffenburg.

Deppermann, Arnulf, \& Günthner, Susanne (2015) (Hrsg.): Temporality in interaction. Amsterdam: Benjamins.

Diessel, Holger (2004): The acquisition of complex sentences. Cambridge: Cambridge University Press.

Du Bois, John W. (2014): Towards a dialogic syntax. Cognitive Linguistics 25 (3), 359-410.

Evans, Nicholas (2007): Insubordination and its uses. In Irina Nikolaeva (Hrsg.), Finitness: Theoretical and empirical foundations, 366-431. Amsterdam: Benjamins.

Fauconnier, Gilles (1985): Mental spaces. Cambridge: MIT Press.

Feilke, Helmuth \& Angelika Linke (2009): Oberfläche und Performanz - Zur Einleitung. In Helmuth Feilke \& Angelika Linke (Hrsg.), Oberfläche und Performanz. Untersuchungen zur Sprache als dynamischer Gestalt, 3-17. Tübingen: Niemeyer.

Fischer, Kerstin (2015): Conversation, construction grammar, and cognition. Language and Cognition 7, 563-588.

Givón, Talmy (2009): Multiple routes to clause union: The diachrony of complex verb phrases. In Talmy Givón \& Masayoshi Shibatani (Hrsg.), Syntactic complexity: Diachrony, acquisition, neurocognition, and evolution, 81-118. Amsterdam: Benjamins.

Gumperz, John J. (1982): Discourse strategies. Cambridge: Cambridge University Press.

Günthner, Susanne (1999): Wenn-Sätze im Vor-Vorfeld: Ihre Formen und Funktionen in der gesprochenen Sprache. Deutsche Sprache 3, 209-235.

Günthner, Susanne (2008a): ,Die Sache ist . . . ': eine Projektorkonstruktion im gesprochenen Deutsch. Zeitschrift für Sprachwissenschaft 27 (1), 39-72.

Günthner, Susanne (2008b): Projektorkonstruktionen im Gespräch: Pseudoclefts, die Sache ist-Konstruktionen und Extrapositionen mit es. Gesprächsforschung - Online-Zeitschrift zur verbalen Interaktion 9, 86-114. http://www.gespraechsforschung-ozs.de (3. Juli 2018).

Günthner, Susanne (2009): Konstruktionen in der kommunikativen Praxis. Zur Notwendigkeit einer interaktionalen Anreicherung konstruktionsgrammatischer Ansätze. Zeitschrift für germanistische Linguistik 37 (3), 402-426.

Günthner, Susanne (2011a): Between emergence and sedimentation. Projecting constructions in German interactions. In Peter Auer \& Stefan Pfänder (Hrsg.), Constructions: Emerging and emergent, 156-185. Berlin u. a.: De Gruyter.

Günthner, Susanne (2011b): Aspekte einer Theorie der gesprochenen Sprache - Plädoyer für eine praxisorientierte Grammatikbetrachtung. In Jan Claas Freienstein, Jan Hagemann \& 
Sven Staffeldt (Hrsg.), Äußern und Bedeuten. Festschrift für Eckard Rolf, 231-250. Tübingen: Stauffenburg.

Günthner, Susanne (2012): Die Schriftsprache als Leitvarietät - die gesprochene Sprache als Abweichung?,Normwidrige‘ wenn-Sätze im Gebrauch. In Susanne Günthner, Wolfgang Imo, Dorothee Meer \& Jan Georg Schneider (Hrsg.), Kommunikation und Öffentlichkeit: Sprachwissenschaftliche Potenziale zwischen Empirie und Norm, 61-84. Berlin u. a.: De Gruyter.

Günthner, Susanne (2015a): ,Geteilte Syntax': Kollaborativ erzeugte dass-Konstruktionen. In Alexander Ziem \& Alexander Lasch (Hrsg.), Konstruktionsgrammatik IV. Konstruktionen als soziale Konventionen und kognitive Routinen, 25-40. Tübingen: Stauffenburg.

Günthner, Susanne (2015b): Ko-Konstruktionen im Gespräch: Zwischen Kollaboration und Konfrontation. In Ulrich Dausendschön-Gay, Elisabeth Gülich \& Ulrich Krafft (Hrsg.), KoKonstruktionen in der Interaktion. Die gemeinsame Arbeit an Äußerungen und anderen sozialen Ereignissen, 55-74. Bielefeld: transcript Verlag.

Günthner, Susanne (2015c): Grammatische Konstruktionen im Kontext sequenzieller Praktiken -,was heißt $x^{6}$-Konstruktionen im gesprochenen Deutsch. In Jörg Bücker, Susanne Günthner \& Wolfgang Imo (Hrsg.), Konstruktionsgrammatik V: Konstruktionen im Spannungsfeld von sequenziellen Mustern, kommunikativen Gattungen und Textsorten, 187-218. Tübingen: Stauffenburg.

Günthner, Susanne (2017): Alleinstehende Nebensätze: Insubordinierte wenn-Konstruktionen in der kommunikativen Praxis. In Yüksel Ekinci, Elke Montanari \& Lirim Selmani (Hrsg.), Grammatik und Variation. Festschrift für Ludger Hoffmann zum 65. Geburtstag, 97-110. Heidelberg: Synchron.

Günthner, Susanne (2020): Practices of clause-combining: From complex wenn-constructions to insubordinate conditionals in everyday spoken German. In Yael Maschler, Simona Pekarek Doehler, Jan Lindström \& Leelo Keevallik (Hrsg.), Emergent syntax for conversation: Clausal patterns and the organization of action, 185-219. Amsterdam/New York: Benjamins.

Günthner, Susanne \& Wolfgang Imo (2006) (Hrsg.): Konstruktionen in der Interaktion. Berlin u. a.: De Gruyter.

Günthner, Susanne \& Paul Hopper (2010): Zeitlichkeit \& sprachliche Strukturen: Pseudoclefts im Englischen und Deutschen. Gesprächsforschung - Online-Zeitschrift zur verbalen Interaktion 11, 1-28. http://www.gespraechsforschung-ozs.de (3. Juli 2018).

Günthner, Susanne, Wolfgang Imo \& Jörg Bücker (2014) (Hrsg.): Grammar and dialogism. Sequential, syntactic, and prosodic patterns between emergence and sedimentation. Berlin u. a.: De Gruyter.

Helbig, Gerhard \& Joachim Buscha (2005): Deutsche Grammatik. Ein Handbuch für den Ausländerunterricht. Berlin u. a.: Langenscheidt.

Hilpert, Martin (2015): Kollaborative Insubordination in gesprochenem Englisch: Konstruktion oder Umgang mit Konstruktionen? In Alexander Ziem \& Alexander Lasch (Hrsg.), Konstruktionsgrammatik IV. Konstruktionen als soziale Konventionen und kognitive Routinen, 25-40. Tübingen: Stauffenburg.

Hoffmann, Ludger (2013): Deutsche Grammatik. Berlin: Erich Schmidt. Hopper, Paul (1987): Emergent grammar. Berkeley Linguistic Society 13, 139-157. Hopper, Paul (1988): Emergent Grammar and the a priori grammar postulate. In Deborah Tannen (Hrsg.), Linguistics in context, 117-133. Norwood: Ablex. 
Hopper, Paul (2004): The openness of grammatical constructions. Chicago Linguistic Society 40, 153-175.

Hopper, Paul (2011): Emergent grammar and temporality in interactional linguistics. In Peter Auer \& Stefan Pfänder (Hrsg.), Constructions: Emerging and emergent, 22-44. Berlin u. a.: De Gruyter.

Hopper, Paul \& Elizabeth Traugott (1993/2003): Grammaticalization. Cambridge: Cambridge University Press.

Hopper, Paul \& Sandra A. Thompson (2008): Projectability and clause combining in interaction. In Ritva Laury (Hrsg.), Crosslinguistic studies of clause combining. The multifunctionality of conjunctions, 99-124. Amsterdam: Benjamins.

Imo, Wolfgang (2007): Construction Grammar und Gesprochene-Sprache-Forschung: Konstruktionen mit zehn matrixsatzfähigen Verben im gesprochenen Deutsch. Tübingen: Niemeyer.

Imo, Wolfgang (2011): Where Does the Mountain Stop? A Granular Approach to the Concept of Constructions-as-Signs. In Stefan Engelberg, Anke Holler \& Kristel Proost (Hrsg.), Sprachliches Wissen zwischen Lexikon und Grammatik, 113-148. Berlin u. a.: De Gruyter.

Imo, Wolfgang (2014): Elliptical structures as dialogical resources for the management of understanding. In Susanne Günthner, Wolfgang Imo \& Jörg Bücker (Hrsg.), Grammar and dialogism. Sequential, syntactic, and prosodic patterns between emergence and sedimentation, 139-176. Berlin u. a.: De Gruyter.

Jespersen, Otto (1924): The philosophy of grammar. London: George Allen \& Unwin.

Johnson-Laird, Philipp N. (1986): Conditionals and mental models. In Elizabeth Traugott, Alice ter Meulen, Judy S. Reilly \& Charles A. Ferguson (Hrsg.), On conditionals, 55-75. Cambridge: Cambridge University Press.

Köpcke, Klaus-Michael \& Klaus-Uwe Panther (1989): On correlations between word order and pragmatic function of conditional sentences in German. Journal of Pragmatics 13, $685-711$.

Langacker, Ronald W. (1987): Foundations of cognitive grammar. Stanford: Stanford University Press.

Langacker, Ronald W. (2008): Cognitive grammar. A basic introduction. Oxford: Oxford University Press.

Linell, Per (2005): The written language bias. London: Routledge.

Linell, Per (2009): Rethinking language, mind, and world dialogically: Interactional and contextual theories of human sense-making. Charlotte: Information Age Pub.

Luckmann, Thomas (2002): Wissen und Gesellschaft. Ausgewählte Aufsätze. Konstanz: UVK.

Ono, Tsuyoshi, \& Sandra A. Thompson (1995): What can conversation tell us about syntax? In Philip W. Davis (Hrsg.), Alternative linguistics: Descriptive and theoretical modes, 213-271. Amsterdam: Benjamins.

Oppenrieder, Wilhelm (1989): Selbständige Verb-Letzt-Sätze: Ihr Platz im Satzmodussystem und ihre intonatorische Kennzeichnung. In Hans Altmann, Anton Batliner \& Wilhelm Oppenrieder (Hrsg.), Zur Intonation von Modus und Fokus im Deutschen, 163-244. Tübingen: Niemeyer.

Pekarek Doehler, Simona (2011): Clause-combining and the sequencing of actions: Projector constructions in French talk-in-interaction. In Ritva Laury \& Ryoko Suzuki (Hrsg.), Subordination in conversation. A cross-linguistic perspective, 103-148. Amsterdam: Benjamins.

Riesel, Elise (1964): Der Stil der deutschen Alltagsrede. Leipzig: Reclam. 
Scholz, Ulrike (1991): Wunschsätze im Deutschen - Formale und funktionale Beschreibung. Satztypen mit Verberst- und Verbletztstellung. Tübingen: Niemeyer.

Schütz, Alfred (1932/1973): Der sinnhafte Aufbau der sozialen Welt: Eine Einleitung in die verstehende Soziologie. Wien: Julius Springer.

Selting, Margret, Auer, Peter, Barth-Weingarten, Dagmar, Bergmann, Jörg, Bergmann, Pia, Birkner, Karin, Couper-Kuhlen, Elizabeth, Deppermann, Arnulf, Gilles, Peter, Günthner, Susanne, Hartung, Martin \& Kern, Friederike (2009): Gesprächsanalytisches Transkriptionssystem 2 (GAT 2). Gesprächsforschung - Online-Zeitschrift zur verbalen Interaktion 10, 353-402. http://www.gespraechsforschung-ozs.de/ (3. Juli 2018).

Sweetser, Eve Eliot (1990): From etymology to pragmatics. Metaphorical and cultural aspects of semantic structure. Cambridge: Cambridge University Press.

Szczepek, Beatrice (2000a): Formal aspects of collaborative productions in English conversation. InLiSt: Interaction and Linguistic Structures 17. http://www.inlist.uni-bay reuth.de/ (3. Juli 2018).

Szczepek, Beatrice (2000b): Functional aspects of collaborative productions in English conversation. InLiSt: Interaction and Linguistic Structures 21. http://www.inlist.uni-bay reuth.de/ (3. Juli 2018).

Tomasello, Michael (2005): Constructing a language. Cambridge: Harvard University Press.

Tomasello, Michael (2006): Konstruktionsgrammatik und früher Erstspracherwerb. In Kerstin Fischer \& Anatol Stefanowitsch (Hrsg.), Konstruktionsgrammatik: Von der Anwendung zur Theorie, 19-38. Tübingen: Stauffenburg.

Wegner, Lars (2010): Unverbundene WENN-Sätze in der gesprochenen Sprache - zur zeitlichen Emergenz syntaktischer Konstruktionen im Interaktionsprozess. GIDIArbeitspapierreihe 28 (12). http://noam.uni-muenster.de/gidi/arbeitspapiere/arbeitspapier28.pdf (3. Juli 2018).

Weinrich, Harald (1993/2007): Textgrammatik der deutschen Sprache. Hildesheim: Georg Olms Verlag.

Weuster, Edith (1983): Nicht-eingebettene Satztypen mit Verb-Endstellung im Deutschen. In Klaus Olszok \& Edith Weuster (Hrsg.), Zur Wortstellungsproblematik im Deutschen, 7-88. Tübingen: Narr.

Zifonun, Gisela et al. (1997): Grammatik der deutschen Sprache. Bd. 1-3. Berlin u. a.: De Gruyter. 